\title{
Temporary Filters and Liver Mobilization Technique Improve the Safety and Prognosis of Radical Nephrectomy and Inferior Vena Cava Thrombectomy in Renal Cell Carcinoma with Subdiaphragmatic Thrombosis
}

\author{
Jian-ping Zhang Yu Zhu Yu-jun Liu Li Zhang Li-an Sun Jian-ming Guo \\ Zong-ming Lin \\ Department of Urology, Zhongshan Hospital, Fudan University, Shanghai, PR China
}

\section{Key Words}

Temporary filters $\cdot$ Renal cell carcinoma $\cdot$ Liver mobilization technique Subdiaphragmatic thrombosis a liver mobilization technique to expose the IVC can effectively prevent tumor thrombosis embolism shedding and improve surgical safety.

\begin{abstract}
Aims: This study aimed to investigate the safety and efficacy of preoperative temporary inferior vena cava (IVC) filter placement and intraoperative application of a liver mobilization technique. Materials and Methods: The experiment cohort of 42 cases and the control cohort of 36 cases of renal cell carcinoma involving the IVC were analyzed retrospectively. In the experiment cohort, patients were implanted with a temporary IVC filter as routine preoperative treatment. The control cohort of 36 cases received traditional radical nephrectomy + IVC thrombectomy. Results: In the experiment cohort, 42 cases did not show any symptom of tumor thrombus embolism perioperatively. The average operation time was $220 \mathrm{~min}$ and the average blood loss was $750 \mathrm{ml}$. Overall survival rate of improved surgery was significantly higher than traditional surgery $(p=0.0055)$. Moreover, tumor thrombus size and position was associated with overall survival $(p=0.0185)$. Conclusions: Preoperative temporary IVC filter placement and intraoperative application of
\end{abstract}

\section{Introduction}

Renal cell carcinoma (RCC) frequently invades the inferior vena cava (IVC) to form a thrombus [1-4]. IVC thrombus has been used as a sign of poor prognosis or even a contraindication for surgery of RCC. However, with the development of surgical techniques, it has become possible to perform thrombus removal. For RCC patients without distal metastases, complete tumor resection and tumor thrombus removal by surgery have shown a good efficacy with a 5-year survival rate of 45-69\% [1$4]$. Even for cases with distal tumor metastases, surgical treatment could still promote the survival rate and improve quality of life.

However, IVC thrombectomy is a highly risky surgery that may lead to a variety of dangerous complications, including heart and pulmonary embolism caused by intraoperative tumor thrombus shedding, inter- and post-

\section{KARGER}

E-Mail karger@karger.com

www.karger.com/uin
(C) 2013 S. Karger AG, Basel

0042-1138/13/0913-0279\$38.00/0
Zong-ming Lin

Department of Urology, Zhongshan Hospital

Fudan University

Shanghai 200032 (PR China)

E-Mail zongminglin@163.com 
operative bleeding, and postoperative dysfunction of the liver and kidney. These complications have a high occurrence rate and subsequently a high mortality rate $[5,6]$. During surgery, it is crucial to separate and control the IVC to avoid tumor thrombosis embolism shedding. Although it is difficult to expose the hepatic IVC, a surgical technique to isolate the liver has been used to expose the hepatic IVC [7]. The temporary IVC filter can be used to prevent the shed tumor thrombus from entering the blood, thus avoiding dangerous complications such as pulmonary embolism. In this study we aimed to investigate the safety and efficacy of radical nephrectomy + IVC thrombectomy, and evaluate the efficacy of preoperative temporary IVC filter placement and intraoperative application of a liver mobilization technique to expose the IVC. To facilitate the determination of the surgery plan, we used preoperative CT or MRI to assess the level of the tumor thrombus. Our results showed that preoperative temporary IVC filter placement and intraoperative application of the liver-harvesting surgical technique to expose the IVC could effectively prevent tumor thrombosis embolism shedding and improve surgical safety.

\section{Material and Methods}

\section{Clinical Data Source}

The experiment cohort of 42 cases (January 2003 to December 2007, Department of Urology, Zhongshan Hospital) of RCC involving the IVC were analyzed retrospectively. All of them received improved surgery which used temporary filters and a liver mobilization technique to improve the safety of nephrectomy + IVC thrombectomy. 36 cases who received traditional nephrectomy + IVC thrombectomy were used as a control cohort in survival analysis. All patients received preoperative CT or MRI to assess the level of the IVC thrombus. The study protocols were approved by the institutional review board of Zhongshan Hospital and all patients gave informed consent.

\section{Study Group Design}

The experiment cohort which received improved surgery was divided into three groups according to the location of the tumor thrombus: (1) group A, the tumor thrombus was $<2 \mathrm{~cm}$ above the upper edge of the renal vein; (2) group B, the tumor thrombus was below the opening of the hepatic vein, and (3) group $\mathrm{C}$, the tumor thrombus was at or above the hepatic vein but lower than the diaphragm. All cases with tumor thrombus above the diaphragm or involving the right atrium were excluded. The basic clinical data are listed in table 1.

In the experiment cohort, preoperative routine examination revealed 5 cases of distal metastases, including 4 cases of lung metastases and 1 case of liver metastases. Although these patients had severe symptoms including hematuria, back pain, anemia, or abnormal liver function, they were treated by surgery to improve quality of life and implanted with a temporary IVC filter as routine
Table 1. Clinical characteristics of 42 patients in the experiment cohort

\begin{tabular}{lllll}
\hline Clinical factors & Total cases & Group A & Group B & Group C \\
\hline Patients, n & 42 & 11 & 19 & 12 \\
Average age, years & 61 & 60 & 63 & 58 \\
$\begin{array}{l}\text { Gender, n } \\
\quad \text { Male }\end{array}$ & 26 & 8 & 11 & 7 \\
$\quad$ Female & 16 & 3 & 8 & 5 \\
Tumor location, n & & & & \\
$\quad$ Right & 31 & 4 & 17 & 10 \\
$\quad 11$ & 7 & 2 & 2 \\
$\quad$ Left & 10.6 & 9.5 & 10.1 & 12.6 \\
$\begin{array}{l}\text { Rumor size, cm } \\
\text { Renal hilum lymph }\end{array} \quad$ & & & \\
$\quad$ node metastasis, n & 7 & 1 & 2 & 4 \\
$\begin{array}{l}\text { Distal metastasis, n } \\
\text { Histological type, n }\end{array}$ & 5 & 1 & 1 & 3 \\
$\quad$ Clear cell type & 32 & 9 & 14 & 9 \\
$\quad$ Other types & 10 & 2 & 5 & 3 \\
\hline
\end{tabular}

preoperative treatment. For those patients with tumor thrombi behind the liver, the liver-harvesting surgical technique was applied to separate and turn the liver to the left to expose the IVC, and then the blood flow was blocked to accomplish the IVC thrombectomy. The filter was removed postoperatively on the same day. A routine follow-up study was performed.

\section{Results}

\section{Preoperative Preparation: Placement of Temporary IVC Filter}

All patients in the experiment cohort received temporary filter placement on or 1 day prior to surgery. The temporary filter was placed by a physician from either the Invasive Technology Department or the Vascular Surgery Department. Tempofilter II ${ }^{\mathrm{TM}}$ (B. Braun, Melsungen, Germany) implantation was preferentially performed according to the Seldinger technique by the percutaneous route through the right internal jugular vein in 36 cases (fig. 1), and an OPTEASE ${ }^{\circledR}$ vena cava filter (Cordis, Miami Lakes, Fla., USA) was placed through the reversed route of the femoral vein in the other 6 cases. Preimplantation cavography was performed to assess the features of intracaval thrombus. After the guidewire was threaded into the IVC, the subcutaneous puncture site was enlarged and a pocket of approximately $20 \mathrm{~mm}$ was created for the anchoring device. The dilator and sheath were inserted into the IVC along the guidewire under fluoroscopic guidance. Iodinated contrast agent was in- 
Fig. 1. Temporary IVC filter placement. a MRI showing right RRC, IVC thrombus behind the liver. b Temporary IVC filter was placed through the jugular vein.
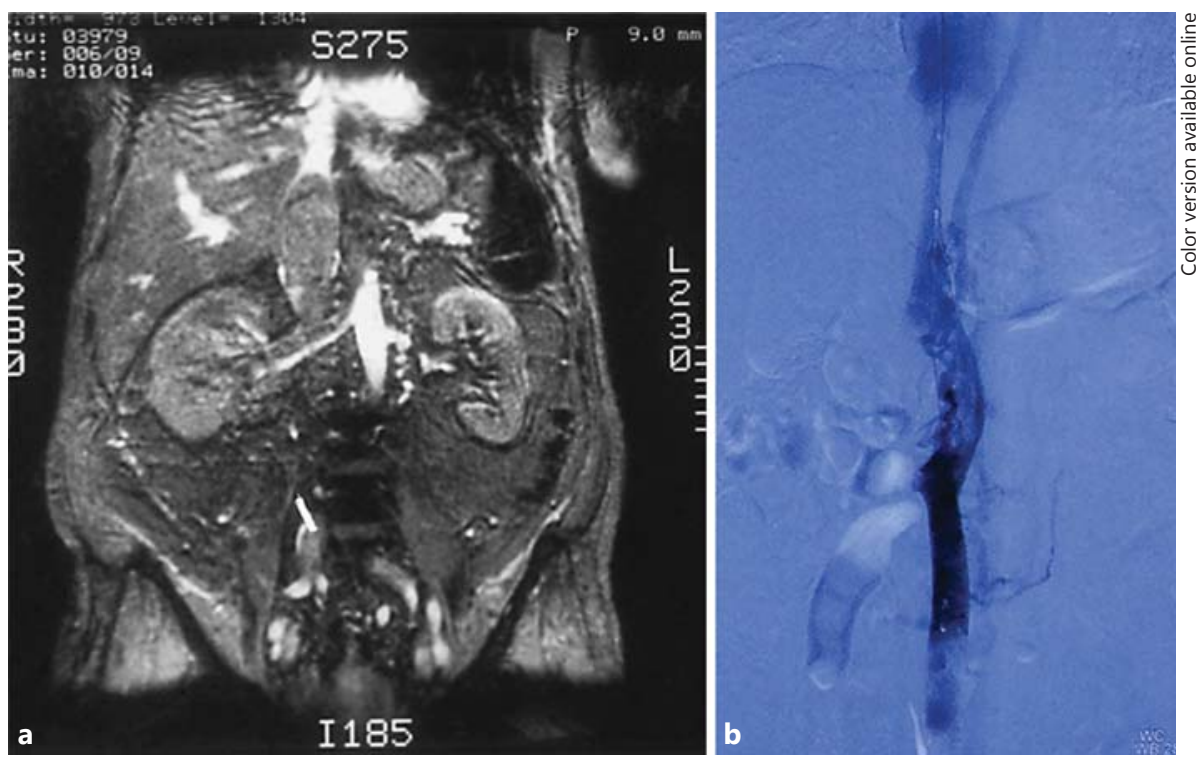

jected to locate the uppermost extension of the IVC thrombus. After the dilator was removed, a syringe containing the filter was screwed onto the proximal end of the sheath. After being pushed to the distal end of the sheath by using the tethering catheter, the filter was deployed above the thrombus by removing the sheath. Next, the anchoring device was attached to the tethering catheter after checking the position of the filter, and the excess catheter was sectioned immediately above the button. Retrieval of the temporary filter was performed after Doppler sonography to exclude the large thrombus $(>1 \mathrm{~cm})$ in the filter on the day of surgery. After the anchoring device was located by palpation, a short incision was made to release the anchoring device. The catheter and filter were removed simply by pulling the tethering catheter smoothly outward.

\section{Liver Mobilization Technique in Radical}

Nephrectomy + IVC Thrombectomy

After anesthesia, the supine patient received an ipsilateral elevation of $\sim 30^{\circ}$. An incision was made at the rib and extended ipsilaterally to the anterior axillary line and contralaterally to the mid-clavicular line. In some patients, the incision was extended to the xiphoid to expose the diaphragm and to isolate the IVC. The kidney and tumor were isolated and removed. The cut ends of the veins were temporarily ligated and the lymph nodes near the kidney door were maximally removed to expose the cut end of the renal vein. The IVC, starting from $3 \mathrm{~cm}$ below the renal vein opening to the head of the tumor thrombus, was isolated and the contralateral renal vein was isolated along the free edge of the IVC.

In group A patients, pulling the liver to the diaphragm was sufficient to isolate the IVC, whereas in group B and $\mathrm{C}$ patients, to expose the hepatic IVC and hepatic vein, it was necessary to cut off the round ligament of the liver, the left and right triangular ligaments, and the sagittal and coronal ligaments, and isolate and pull the liver to the lower left (fig. 2). For group B patients, the IVC could be blocked below the hepatic vein. However, for group C patients, to isolate sufficient IVC, the short hepatic veins should be cut off and the sheath of the IVC near the diaphragm foot should be opened. Meanwhile, to avoid the blockade of hepatic blood flow, the tumor thrombus could be gently pushed to below the hepatic vein and, if necessary, the blood flow in the hepatoduodenal ligament should be blocked. The proximal and distal IVC were blocked sequentially. In group $\mathrm{C}$ patients, if the tumor thrombus could not be pushed to below the hepatic vein, the hepatoduodenal ligament should be blocked using the Pringle maneuver method. The contralateral renal vein was blocked using the same method. A ring-shaped incision was made at the opening of the renal vein to open the IVC and extended upward to the head of the tumor thrombus to isolate and remove the whole tumor thrombus. After being rinsed with heparin saline, the IVC was continuously sutured. After the blockade was removed in a reversed order to allow blood flow, the aorta and the IVC lymph nodes were re-cleaned, and the cut end of the renal artery was re-sutured. 
Fig. 2. Liver mobilization technique in nephrectomy + IVC thrombectomy. a Sagittal ligament and coronary ligament of the liver were cut. $\mathbf{b}$ The liver was isolated to expose IVC.

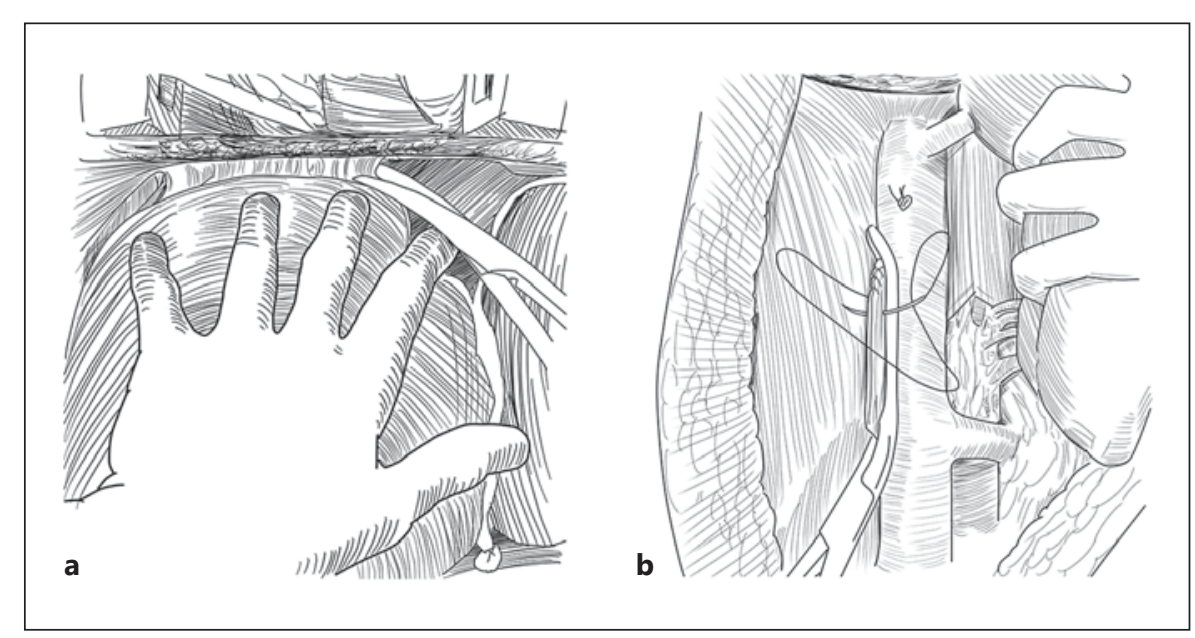

Table 2. Perioperative data for 42 patients in the experiment cohort

\begin{tabular}{|c|c|c|c|c|}
\hline Variable & Total cases & Group A & Group B & Group C \\
\hline Patients, n & 42 & 11 & 19 & 12 \\
\hline Surgery time, $\min$ & 220 & 170 & 230 & 250 \\
\hline $\begin{array}{l}\text { Blood loss, ml } \\
\text { Cases of }\end{array}$ & 750 & 400 & 800 & 1,000 \\
\hline transfusion, $\mathrm{n}$ & 12 & 2 & 5 & 5 \\
\hline $\begin{array}{l}\text { Blood volume, } \mathrm{ml} \\
\text { Postoperative }\end{array}$ & 800 & $400-800$ & $600-1,200$ & $1,000-2,000$ \\
\hline metastasis, $\mathrm{n}$ & $15 / 37$ & $3 / 10$ & $7 / 18$ & $5 / 9$ \\
\hline
\end{tabular}

One month after surgery, the first follow-up was performed including chest $\mathrm{x}$-ray, abdominal type-B ultrasonic, and liver and kidney function examination. Since then, the follow-up was performed every 3 months, and the abdominal CT was performed every 3 months. In the case that the chest $\mathrm{x}$-ray was abnormal, a chest CT was performed. The follow-up was 5 years for all patients.

\section{Improved Surgical Technique in Radical \\ Nephrectomy + IVC Thrombectomy Ameliorate \\ Patients' Perioperative Status}

The perioperative data of the experiment cohort are listed in table 2. For surgery time, it was the shortest in group A and showed no significant difference in groups $\mathrm{B}$ and $\mathrm{C}$, which was probably because groups $\mathrm{B}$ and $\mathrm{C}$ required free livers and had wider ranges of surgical areas and subsequently higher amounts of bleeding. None of the 42 patients showed inter- or postsurgical pulmonary embolism, but 1 patient in group B showed severe lung infection 3 days after surgery and died after 2 weeks. In group C, 4 patients received downward thrombosis extrusion to avoid the blockade of the hepatic blood flow and 8 cases received hepatic blood flow blockade at the opening of the hepatic vein. Of the 8 patients, 3 cases showed liver dysfunction such as elevated transaminase level and moderate jaundice, which were relieved after treatment at the Internal Medicine Department. None of the 42 patients showed obvious renal dysfunction or untreatable lower extremity edema.

\section{Temporary Filters and Liver Mobilization Technique}

Cooperate to Improve Prognosis of RCC Patients with

Subdiaphragmatic Thrombosis

During the 5-year follow-up study, metastases developed in 15 of the 37 patients (40.6\%) without preoperative distal metastases, including 11 cases of lung metastases, 1 case of bone metastases, 1 case of liver metastases, 1 case of contralateral adrenal metastases, and 1 case of multiple metastases. Among 15 patients, group $C$ showed the highest proportion of metastases, $5 / 9$ (55\%). For 5 patients with preoperative distal metastases, the symptoms of hematuria and low back pain were significantly improved, and the median survival time was 14 (4-38) months.

Kaplan-Meier survival analysis indicated that the overall survival rate of 42 RCC patients with improved surgery was significantly higher than 36 patients with traditional surgery ( $p=0.0055$; fig. $3 \mathrm{a}$ ). Moreover, the overall survival for patients in group A (the tumor thrombus was $<2 \mathrm{~cm}$ above the upper edge of the renal vein) and group B (the tumor thrombus was below the opening of the hepatic vein) were significantly higher than group C (the tumor thrombus was at or above the hepatic vein but 


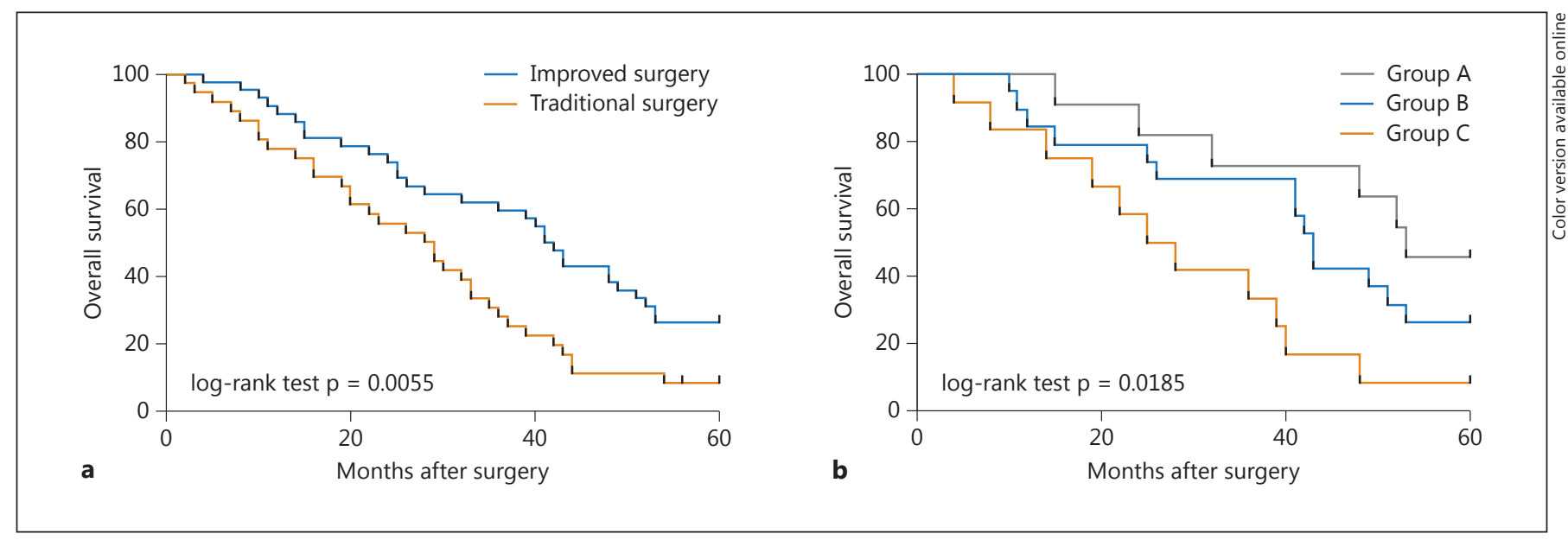

Fig. 3. Overall survival in RCC patients with improved and traditional surgery. a Kaplan-Meier survival analysis of 42 cases in the experiment cohort who received improved surgery and 36 cases in the control cohort who received traditional surgery. b Kaplan-
Meier survival analysis of three groups with different tumor thrombus size and position in the experiment cohort. $\mathrm{p}$ value was calculated by log-rank test. lower than the diaphragm) ( $\mathrm{p}=0.0185$; fig. 3b). These results indicate that tumor thrombus size and position is associated with overall survival.

\section{Discussion}

For patients with RCC + IVC thrombus, the combined therapy of radical nephrectomy + IVC thrombectomy is the only possible approach to cure them $[3,4]$. However, surgery has a high risk of dangerous complications, especially tumor thrombosis embolism shedding, which is rare $(2-4 \%)$ but has a high mortality rate of $75 \%[2,5]$. Consequently, it is necessary to take a series of measures to avoid tumor thrombosis embolism shedding $[6,8]$.

To facilitate the determination of the surgery plan, it is very important to use preoperative CT or MRI to access the level of the tumor thrombus. Whether the position of the tumor thrombus can affect the prognosis remains a subject of debate $[2,9]$. However, tumor thrombi at high positions (e.g. thrombus above the hepatic vein or invading into the right atrium) always require complex surgical operations such as hypothermic cardiopulmonary bypass, cardiac arrest, or thrombus monitoring by transesophageal echocardiography, which can cause severe complications $[6,10,11]$. Furthermore, a number of studies have suggested that patients with tumor thrombus above the diaphragm showed a poor prognosis and a higher rate of metastases, probably because metastases could have existed preoperatively $[6,12]$. Thus we always first perform kidney tumor removal for patients with tumor thrombus above the diaphragm and then apply the targeted therapy or immunotherapy.

Temporary IVC filter placement is a feasible method to avoid tumor thrombosis embolism shedding. The IVC filter was initially used for the prevention of pulmonary embolism caused by lower limb deep vein thrombosis $[13,14]$. During the process of radical nephrectomy + IVC thrombectomy, the filter can be used to arrest the shedding of IVC thrombus [15]. Thus the filter should be placed through the route of the superior vena cava, otherwise thrombus shedding could occur. In our study, all 42 patients received filter placement preferentially through the superior vena cava route, 36 cases were successful and the other 6 cases were changed to be placed through a reversed route of the femoral vein. Before the placement, the position of the tumor thrombus should be determined through imaging approaches, and the filter should be placed $1-2 \mathrm{~cm}$ apart from the head of the thrombus to facilitate the blockade of the IVC. The temporary filter can be removed shortly after surgery, and thus can avoid the re-formation of the thrombus on the filter in patients in a hypercoagulable state and avoid the use of anticoagulant drugs.

During the process of surgery, surgeons should avoid extruding the tumor thrombus before blocking the head of the IVC. If the head of the tumor thrombus is below the liver it would be easy to control the IVC, but if the tumor 
thrombus is behind the liver it would be difficult to expose the IVC. In this situation, the primary tumor usually is so large that the space for surgery is limited [12]. We chose to gently isolate the kidney and tumor and remove them. Next we applied a liver-harvesting surgical technique to isolate the liver and, if necessary (i.e. tumor thrombus is above the hepatic vein), isolated and cut off the short hepatic veins. We then pulled the liver to the lower left so that the full length of the IVC could be exposed. In this way, we could provide sufficient space for surgery [7]. In the case that the tumor thrombus is above the hepatic vein, blockade of the hepatic blood flow should be avoided. To prevent blockade of the hepatic blood flow, surgeons can gently push the head of the tumor thrombus to below the hepatic vein after the IVC is exposed.
After a 5-year follow-up, we found that the overall survival rate of improved surgery was significantly higher than traditional surgery $(\mathrm{p}=0.0055)$. Moreover, tumor thrombus size and position was associated with overall survival ( $\mathrm{p}=0.0185)$. For RCC patients with IVC thrombus but no distal metastasis, treatment with surgery can achieve a good efficacy. To effectively avoid tumor thrombosis embolism shedding, surgeons should: (1) assess the preoperative level of the tumor thrombus; (2) make use of a temporary IVC filter, and (3) avoid the extrusion of the tumor thrombus before controlling the proximal IVC. To fully expose the IVC to achieve sufficient surgery space, surgeons can first remove the kidney and the liver, and then apply a liver-harvesting surgical technique to isolate liver.

\section{References}

-1 Martinez-Salamanca JI, Huang WC, Millan I, et al: Prognostic impact of the 2009 UICC/ AJCC TNM staging system for renal cell carcinoma with venous extension. Eur Urol 2011;59:120-127.

-2 Bertini R, Roscigno M, Freschi M, et al: Impact of venous tumour thrombus consistency (solid vs. friable) on cancer-specific survival in patients with renal cell carcinoma. Eur Urol 2011;60:358-365.

3 Ciancio G, Manoharan M, Katkoori D, et al: Long-term survival in patients undergoing radical nephrectomy and inferior vena cava thrombectomy: single-center experience. Eur Urol 2010;57:667-672.

4 Takeda H, Nakano Y, Kashiwagi Y, Yoshino Y, Gotoh M: Downsizing a thrombus of advanced renal cell carcinoma in a presurgical setting with sorafenib. Urol Int 2012;88:235-237.

$\checkmark 5$ Galvez JA, Clebone A, Garwood S, Popescu WM: Fatal intraoperative cardiac thrombosis in a patient with renal cell carcinoma. Anesthesiology 2011;114:1212.
6 Shuch B, Larochelle JC, Onyia T, et al: Intraoperative thrombus embolization during nephrectomy and tumor thrombectomy: critical analysis of the University of California-Los Angeles experience. J Urol 2009;181:492-498.

7 Gallucci M, Borzomati D, Flammia G, et al: Liver harvesting surgical technique for the treatment of retrohepatic caval thrombosis concomitant to renal cell carcinoma: perioperative and long-term results in 15 patients without mortality. Eur Urol 2004;45:194-202.

8 Yang Y, Sun S, Xiao X, et al: Temporary balloon occlusion of inferior vena cava in resection of renal tumor with vena cava thrombus extension. Urology 2009;73:645-648.

$\checkmark 9$ Rigaud J, Hetet JF, Braud G, et al: Surgical care, morbidity, mortality and follow-up after nephrectomy for renal cancer with extension of tumor thrombus into the inferior vena cava: retrospective study since 1990s. Eur Urol 2006;50:302-310.

10 Shirodkar SP, Ciancio G, Soloway MS: Vascular stapling of the inferior vena cava: further refinement of techniques for the excision of extensive renal cell carcinoma with unresectable vena-caval involvement. Urology 2009; 74:846-850.
1 Granberg CF, Boorjian SA, Schaff HV, et al: Surgical management, complications, and outcome of radical nephrectomy with inferior vena cava tumor thrombectomy facilitated by vascular bypass. Urology 2008;72: 148-152.

12 Zini L, Koussa M, Haulon S, et al: Results of endoluminal occlusion of the inferior vena cava during radical nephrectomy and thrombectomy. Eur Urol 2008;54:778-783.

13 Darcy MD, Cardella JF, Hunter DW, et al: Experience with the Amplatz retrievable vena caval filter. Work in progress. Radiology 1986;161:611-614.

14 Cope C, Baum RA, Duszak RA Jr: Temporary use of a bird's nest filter during iliocaval thrombolysis. Radiology 1996;198:765-767.

15 Blute ML, Boorjian SA, Leibovich BC, Lohse CM, Frank I, Karnes RJ: Results of inferior vena caval interruption by greenfield filter, ligation or resection during radical nephrectomy and tumor thrombectomy. J Urol 2007; 178:440-445. 